\title{
RESEARCH AND PRACTICE IN ANESTHESIOLOGY
}

\section{Case Report}

\author{
"Corresponding author \\ Charles Youngblood, MD \\ Assistant Professor \\ Department of Anesthesiology \\ Creighton University Medical Center \\ 601 North $30^{\text {th }}$ Omaha \\ NE 68130, USA \\ Tel. 402-506-2181 \\ E-mail: cfy25655@creighton.edu; \\ CharlesYoungblood@creighton.edu
}

Volume 1 : Issue 1

Article Ref. \#: 1000AOJ1106

\section{Article History}

Received: January $19^{\text {th }}, 2016$

Accepted: June $9^{\text {th }}, 2016$

Published: June $10^{\text {th }}, 2016$

\section{Citation}

Gartner M, Youngblood C. Case report in anesthesiology: essential pulmonary hypertension in a primigravida. Res Pract Anesthesiol Open J. 2016; 1(1): 24-27. doi: 10.17140/ RPAOJ-1-106

\section{Copyright}

(C2016 Youngblood C. This is an open access article distributed under the Creative Commons Attribution 4.0 International License (CC BY 4.0), which permits unrestricted use, distribution, and reproduction in any medium, provided the original work is properly cited.

\section{Case Report in Anesthesiology: Essential Pulmonary Hypertension in a Primigravida}

\author{
Michele Gartner, MS; Charles Youngblood, MD
}

Department of Anesthesiology, Creighton University Medical Center, Omaha, NE 68130, USA

\section{CASE REPORT}

A 21-year old gravida 1, para 0, female with a past medical history of Pulmonary Hypertension $(\mathrm{PH})$ secondary to a congenital heart disease (patent foramen ovale), diaphragmatic hernia status post repair, asthma, and attention-deficit/hyperactivity disorder presented to a tertiary care hospital at 30 weeks gestation for a right heart catheterization. The catheterization was completed without complications and it was found that the patient's Pulmonary Arterial Pressure (PAP) was $54 / 32$ with a mean value of 39 . The PAP was only minimally improved to $46 / 30$ with a mean of 35 after nitric oxide administration.

When the patient presented for follow up, she was complaining of shortness of breath for the last two months and she is unable to walk up a flight of stairs. Due to her history, the patient was admitted to the hospital and was seen by a pulmonologist and an obstetric consultation was ordered. It was determined that the only medications the patient was taking at home were Symbicort, Prenatal Vitamins, Lovenox, Xoprenex, and an iron supplementation. On physical exam, the only pertinent positive was shortness of breath. Her pulmonary history was also significant for a FEVI of $36.6 \%$, and a FEV1/FVC ratio of $62.6 \%$. Given her history of pulmonary arterial hypertension, she was placed on sildenafil $20 \mathrm{mg}$ TID, an epoprostenol drip and lovenox was started after central line placement. An echocardiogram showed an ejection fraction of $60-65 \%$, mild to moderate right ventricular hypertrophy, severe tricuspid valve regurgitation, a right to left shunt and a right ventricular systolic pressure of $104 \mathrm{mmHg}$ (increased from 73 recorded a few weeks prior). Due to her declining right ventricle function, she was started on steroids to induce fetal lung maturity. Over the next few days, the patient developed chest pain, jaw pain, decreased fetal variability, increased oxygen and epoprostenol requirements and hypotension treated with IV fluid boluses. A multidisciplinary care conference including maternal fetal medicine, anesthesia, SICU/PICU surgery team, pulmonary NICU, Labor and Delivery RN, and a pastor was held in order to determine a plan for delivery given the $50 \%$ risk of maternal mortality. It was concluded that the patient would undergo an elective cesarean section and bilateral tubal ligation in two days time.

At the time of delivery, the patient was $315 / 7$ weeks gestation on 8 liters of oxygen and an epoprostenol drip infusing a $14 \mathrm{mg} / \mathrm{kg} / \mathrm{min}$. The morning of surgery, the patient was brought to the preoperative area five hours prior to her surgery. An arterial line was placed in her left dorsalispedis. An epidural was placed at the L3-L4 level without incident. The lidocaine epidural was slowly infused epidural over a period of one hour, mindful to monitor the patient's hemodynamics with the arterial line. The patient was brought to the operating room where she was placed in a supine position with a leftward tilt to avoid inferior vena cava compression. The cesarean section was completed without complications and one dose of methergine was given to control uterine bleeding. Next, a bilateral tubal ligation was carried out without difficulty. Post-partum, the patient did very well. She received dilaudid for pain control and heparin for DVT prophylaxis. Over the next couple of days, her DVT prophylaxis was switched to lovenox, her epoprostenol was increased to $16 \mathrm{mg} / \mathrm{kg} / \mathrm{min}$ and her oxygen requirement dropped to room air at rest, 3 liters on exertion and 1 liter at night. The patient recovered well and was discharged 5 days after delivery. 


\section{RESEARCH AND PRACTICE IN ANESTHESIOLOGY}

A patient with pulmonary arterial hypertension has substantial risks associated with pregnancy. ${ }^{1}$ Pulmonary hypertension is defined by a resting mean pulmonary artery pressure greater than $25 \mathrm{mmHg}$ and a pulmonary capillary wedge pressure less than $15 \mathrm{mmHg}$ in the absence of other causes of precapillary pulmonary hypertension. Clinical classification of $\mathrm{PH}$ has been updated in 2008 and divided into five groups by the World Health Organization (WHO). The groups include pulmonary arterial hypertension (Group 1), pulmonary veno-occlusive disease and/or pulmonary haemangiomatosis (Group 1), $\mathrm{PH}$ due to left heart disease (Group 2), $\mathrm{PH}$ due to lung disease and/or hypoxia (Group 3), Chronic thromboembolic PH (Group 4), and $\mathrm{PH}$ with unclear multifactorial mechanism (Group 5). ${ }^{2}$ Mortality in pregnant women with $\mathrm{PH}$ is has been quoted as $30 \%$ for primary $\mathrm{PH}, 36 \%$ for Eisenmenger's syndrome and 50\% for secondary PH. Despite this improved mortality, recent practice guidelines from the European Society of Cardiology and the American Heart Association/American College of Cardiology strongly discourage pregnancy in patients with $\mathrm{PH}$ and advise termination in early pregnancy. ${ }^{3}$

Pregnancy is heavily discouraged and contraception emphasized due to the difficulty for patients with $\mathrm{PH}$ to cope with the hemodynamic changes related to pregnancy. The changes include increased blood volume, red cell mass, heart rate, cardiac output, left ventricular mass, and decreased pulmonary vascular resistance. The blood volume increases through pregnancy until a plateau occurs between 28 and 34 weeks gestation and remains stable until delivery. The circulating blood volume is increased to $30-50 \%$ above the normal state and red blood cell mass is increased to $25 \%$ above the non-pregnant state. In order to accommodate for these physiological changes, there is a decrease in peripheral vascular resistance. However, patients with pulmonary arterial hypertension are unable to make this adjustment, ${ }^{4}$ restricting the normal progressive increase in pulmonary plasma volume and further stressing the right ventricular load. The drop in systemic vascular resistance increases cardiac output and renal blood flow, which cannot be handled by a failed right ventricle. In addition, in patients with right-to-left shunts, the shunting of blood worsens due to the increased pulmonary pressure, exacerbating hypoxia and pulmonary vasoconstriction.

Furthermore, additional challenges are presented during labor and delivery. During delivery, approximately $500 \mathrm{ml}$ of blood is diverted from the uterine to the material circulation with every uterine contraction, leading to increased cardiac output and venous return. The pain of parturition stimulates the sympathetic nervous system leading to an increase in heart rate, blood pressure and myocardial oxygen consumption, which is further exaggerated during valsalva maneuvers. The pain, acidosis, hypoxia and hypercarbia associated with delivery may increase pulmonary vascular resistance. In addition, acute hypotension from blood loss or vasovagal response during delivery may acutely drop systemic pressure and produce right ventricular ischemia. After delivery, the relief of inferior vena cava compression and auto transfusion from the emptied uterus causes a transient increase in cardiac output and venous return. As a result of the extreme changes to the maternal circulatory system, the highest mortality rate is seen within the first month after delivery.

As of today, there is no consensus on the gold standard management for essential pulmonary hypertension in pregnancy with regards to timing of delivery, choice of delivery technique, and anesthetic choice. Most of the recommended practices are based on case reports and what has been successful for individuals in the past. If a patient with pulmonary hypertension does become pregnant life style changes such as rest, low salt diet and quitting smoking should be implemented. Due to ongoing research, there have been more medications available for the treatment of pulmonary hypertension and aggressive intervention early on may improve outcomes. There are three classes of medication that have entered clinical practice in recent years, resulting in improvements in both functional status and survival. ${ }^{5}$ One class of medication is the endothelial receptor antagonist (bosentan, sitaxsentan, and ambrisentan). The second class of medication is the phosphodiesterase-5 inhibitors (sildenafil and tadalafil). These medications have the convenience of being taken orally and sildenafil received FDA approval for the treatment of pulmonary arterial hypertension in 2005. The third class of medication is prostacyclin/ the prostacyclin analogues (epoprostenol, iloprost, treprostinil). Epoprostenol is given IV, iloprost can be administered IV or nebulized, and treprostinil is given via subcutaneous route. The use of inhaled prostacyclin analogues results in greater concentration of the medication in the pulmonary circulation, and has a theoretical advantage of avoiding some systemic side effects such as hypotension and inhibition of platelet aggregation. Calcium channel blockers are also a treatment option, but should only be used in patients with a functional class II or III in the setting of a positive response to a vasodilation trial. These medications should only be used in a subset of patients and hypotension should be avoided. In addition, a number of case studies report the successful use of nitric oxide around the time of delivery to minimize right ventricular afterload and acutely drop pulmonary vascular resistance.

Even though diuretics are avoided in pregnancy, torsemide or furosemide can be carefully used to manage right heart failure. Antithrombolytic therapy is also recommended for patients with pulmonary arterial hypertension. Warfarin should be avoided between 6 and 12 weeks due to embryonic toxicity. Low molecular weight heparin is the drug of choice since it does not cross the placenta and most physicians will switch to unfractioned heparin near delivery because it is more readily reversible in the event of a hemorrhage.

The mode of delivery is another controversial topic in patients with pulmonary hypertension. The advantages of a vaginal delivery are there are smaller shifts in blood volume, fewer clotting and bleeding complications and a lower risk of infection. However, the disadvantages are a prolonged second stage of labor, uncontrolled vaginal hemorrhage, and adverse hemodynamic effects of the valsalva maneuver. In comparison, the advantages of a caesarian section are it can be performed at 


\section{RESEARCH AND PRACTICE IN ANESTHESIOLOGY}

cians are better able to manage pregnancies complicated by pul-

a suitable time under relative stable conditions, and it can be utilized in urgent deliveries. The disadvantages include increased risk associated with anesthesia, larger shifts in blood volume, more clotting and bleeding complications, higher risk of infection and increased peripheral vascular resistance and right ventricular afterload imparted by positive pressure ventilation in the setting of endotracheal intubation.

A caesarean section is necessary in cases of maternal hemodynamic deterioration or fetal distress requiring emergent delivery. In one study, a planned caesarean section at 34 weeks (the time when blood volume increases by $50 \%$ ) was the preferred method of delivery in stable patients. The justification was a caesarean section can be scheduled during the day with specialists on hand, timing and bimanual compression and suture compression can aid in blood loss. Some studies suggest that if a vaginal delivery is going to be attempted, it should be done in the operating room or in the intensive care unit.

There is no gold standard approach to anesthesia management in a patient with pulmonary arterial hypertension. ${ }^{6}$ During the peripartum period, patients require careful monitoring of oxygen saturation, blood pressure, and heart rhythm. An arterial line and central venous catheter should be placed to monitor blood pressure and right atrial pressure. There is lack of evidence supporting the use of a pulmonary arterial catheter and it is not advised due to its potential complications. During the time of delivery, general anesthesia is mostly avoided. General anesthesia is associated with a higher mortality and it is known to depress cardiac contractility, increase pulmonary vascular resistance, decrease venous return due to positive pressure ventilation, and may result in increased pulmonary arterial resistance during laryngoscope and intubation. ${ }^{7}$ Adequate oxygen, slight hypocapnia, adequate anesthesia level, continuous nitric oxide inhalation and infusion of prostacyclin may all be supportive and help prevent right heart failure caused by the stress of intubation and the operation. After delivery, increasing the nitric oxide dose and maintaining anesthesia with propofol and fentanyl minimizes right ventricular afterload and volume centralization respectively.

Due to the risk associated with general anesthesia, epidural anesthesia or combined spinal anesthesia is recommended. Epidural anesthesia has less effect on cardiac contractility and pulmonary vascular resistance, but the blocks are contraindicated in anticoagulated patients and if used in a caesarean section, it may produce a large decrease in venous return because of the large sympathetic block. Cardiovascular compromise may occur during delivery, which can be treated with dobutamine, norepinephrine or a combination of the two, which provides positive inotropic effects and beneficial right ventricular - pulmonary coupling.

In conclusion, even though the risk of mortality for a patient who becomes pregnant with pulmonary hypertension has decreased, the risk is still substantial enough to advice against it. However, due to further research and medication options physi- monary arterial hypertension. The consensus guidelines recommend the use of epoprostenol and sildenafil to lower pulmonary resistance prior to delivery and aggressive therapy prior to clinical deterioration. This approached worked well for the patient presented. In addition, there is debate over the preferred delivery method as well as the anesthesia management. The patient at hand received a slow infusing epidural and a scheduled caesarean section with arterial line and central line monitoring. The patient and her infant survived the delivery and were discharged home in good condition. Furthermore, the use of lovenox for DVT prophylaxis and unfractioned heparin near the time of delivery was shown to be successful in the case presented. It is unanimously agreed that a multidisciplinary approach is needed to order to properly care for a patient who is pregnant with pulmonary arterial hypertension. Additional research and clinical trials on pulmonary hypertension complicated by pregnancy in needed in order to decrease the risk or mortality since more young women with the disorder are able to reach child bearing age.

\section{CONFLICTS OF INTEREST: None.}

\section{CONSENT}

The patient has provided written permission for publication of the case details.

\section{REFERENCES}

1. Hsu C, Gomberg-Maitland M, Glassner C, Chen J-H. The management of pregnancy and pregnancy-related medical conditions in pulmonary arterial hypertension patients. Int J Clin Pract Suppl. 2011; 65: 6-14. doi: 10.1111/j.1742-1241.2011.02711.x

2. Monagle J, Manikappa S, Ingram B, Malkoutzis V. Pulmonary hypertension and pregnancy: The experience of a tertiary institution over 15 years. Ann Card Anaesth. 2015; 18(2): 153160. doi: 10.4103/0971-9784.154466

3. Bédard E, Dimopoulos K, Gatzoulis M. Has there been any progress made on pregnancy outcomes among women with pulmonary arterial hypertension? Eur Heart J. 2009; 30(3): 256265. doi: 10.1093/eurheartj/ehn597

4. Higton A, Whale C, Musk M, Gabbay E. Pulmonay hypertension in pregnancy: two cases and review of the literature. Intern Med J. 2009; 39(11): 766-770. doi: 10.1111/j.14455994.2009.02051.x

5. Kiely DG, Condliffe R, Webster V, et al. Improved survival in pregnancy and pulmonary hypertension using a multiprofessional approach. BJOG. 2010; 117(5): 565-574. doi: 10.1111/j.14710528.2009.02492.x

6. Paternoster D, Pascoli I, Parotto M, et al. Pulmonary hypertension during pregnancy: management of two cases. Arch Gy- 


\section{RESEARCH AND PRACTICE IN ANESTHESIOLOGY}

necol Obstet. 2010; 281(3): 431-434. doi: 10.1007/s00404-009-

1202-1

7. Lane C, Trow T. Pregnancy and pulmonay hypertension. Clin Chest Med. 2011; 32(1): 165-174. doi: 10.1016/j.ccm.2010. 10.006 